\title{
A Rare Case of Pericardial Decompression Syndrome in a Filipino Female Patient With Suspected Malignant Pericardial Effusion
}

\author{
Danielle Louis E. Villanueva ${ }^{\mathrm{a}, \mathrm{b}}$, Joseph Justin Regalado ${ }^{\mathrm{a}}$, Celia Uy-Agbayani ${ }^{\mathrm{a}}$
}

\begin{abstract}
Pericardial decompression syndrome (PDS) is a rare, under-reported and potentially fatal complication of pericardial drainage characterized by paradoxical hemodynamic deterioration. The onset ranges from immediate to as long as $48 \mathrm{~h}$ post drainage. We present a case of a 51-year-old woman admitted due to progressive dyspnea. She was hemodynamically stable but with signs of cardiac tamponade. On two-dimensional echocardiography (2D-echo) there was a massive pericardial effusion in tamponade physiology. Immediate surgical drainage was done but intra-operatively there was depressed cardiac contractility necessitating inotropic support. Post-operative 2D-echo showed right ventricular (RV) and left ventricular (LV) systolic dysfunction. She was admitted in the intensive care unit (ICU) for $72 \mathrm{~h}$. Repeat 2D-echo showed marked improvement in RV and LV systolic function. She was then discharged improved on the fifth hospital day. The pathophysiology of PDS is still not very clear. The simplest mechanism is that sudden removal of compressing pericardial fluid causes increased venous return with expansion of the RV at the expense of the LV leading to acute heart failure. There are no published studies to propose preventive measures and treatment remains supportive. There has been only one published case reported here in our country. We report this case of a patient who successfully recovered from PDS.
\end{abstract}

Keywords: Pericardial decompression syndrome; Low output cardiac syndrome; Malignant pericardial effusion

\section{Introduction}

Pericardial decompression syndrome (PDS) is a rare but po-

\footnotetext{
Manuscript submitted March 6, 2020, accepted March 23, 2020

aDivision of Cardiovascular Medicine, University of the Philippines, Philippine General Hospital, Manila 1000, Philippines

${ }^{\mathrm{b}}$ Corresponding Author: Danielle Louis E. Villanueva, Division of Cardiovascular Medicine, University of the Philippines, Philippine General Hospital, Taft Avenue, Manila 1000, Philippines. Email: louievillanuevamd@gmail.com
}

doi: https://doi.org/10.14740/jmc3446 tentially fatal complication occurring after pericardial fluid drainage [1]. There is limited epidemiologic data suggesting an overall incidence of less than 5\% [2-6]. We aim to present a successful case of PDS in a patient with suspected malignancy occurring after surgical drainage of pericardial fluid.

\section{Case Report}

We have a 51-year-old patient with a chief complaint of dyspnea. Patient's symptoms started 2 months prior to her admission when she initially noted exertional dyspnea to walking less than $1 \mathrm{~km}$. She also noted intermittent bipedal edema precipitated by prolonged standing and relieved by lying supine. There was progression of her symptoms 1 month after when she complained of two-pillow orthopnea and persistent bipedal edema. These symptoms prompted her to seek consult in our institution.

On consult at the emergency room, her blood pressure was $100 / 60 \mathrm{~mm} \mathrm{Hg}$, heart rate at 94 beats per minute, respiratory rate 22 cycles per minute and temperature at $36.5{ }^{\circ} \mathrm{C}$. There were prominent neck veins with jugular venous pressure $>5$ cm $\mathrm{H}_{2} \mathrm{O}$ but no pulsus paradoxus or Kussmaul's sign was appreciated. Cardiac examination revealed muffled heart sounds with regular rhythm. There were no murmurs or friction rubs heard. Decreased breath sounds and vocal fremitus were noted on the base of the right lung field but no crackles were auscultated. Her abdomen was flat and soft. There was noted grade 1 non-pitting edema on both lower extremities with full peripheral pulses.

Diagnostics done revealed her complete blood count, and renal function tests, liver function tests, thyroid function test, cardiac troponins and serum electrolytes were within acceptable values. Her initial electrocardiogram showed regular sinus rhythm with normal axis at 30 degrees but there were low QRS voltages in all leads. Chest radiograph showed an enlarged heart (cardiothoracic ratio of 0.60 ), with a water bottle configuration, and bilateral pleural effusion with no signs of pulmonary congestion. A transthoracic two-dimensional echocardiography (2D-echo) (Fig. 1) revealed a swinging heart in a massive pericardial effusion with tamponade physiology (early diastolic collapse of the right ventricle (RV) and late inward diastolic collapse of the right atrium with inferior vena cava plethora and respiratory variation of valvular flow upon inspi- 


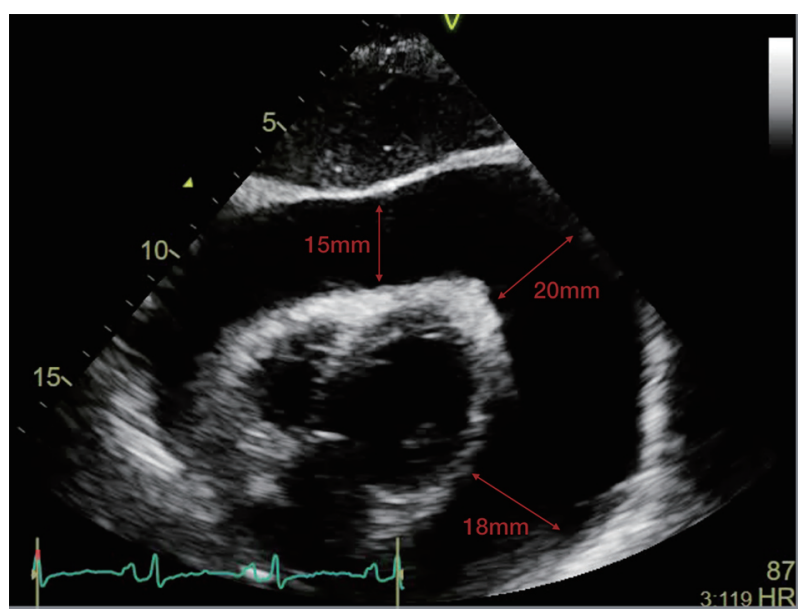

Figure 1. Transthoracic 2D-echo showing massive pericardial effusion (measuring $15 \times 20 \times 18 \mathrm{~mm}$ ) with a "swinging heart". 2D-echo: twodimensional echocardiography.

ration).

The patient was referred to the thoracic and cardiovascular surgery service and was immediately sent to the operating room where she underwent emergency tube pericardiostomy. They drained approximately $1 \mathrm{~L}$ of serous pericardial fluid in $2 \mathrm{~h}$. Intraoperatively, they noted a decrease in cardiac contractility after rapid evacuation of the pericardial fluid. The blood pressure dropped to $70 / 50 \mathrm{~mm} \mathrm{Hg}$ unresponsive to fluid boluses. Inotropic support with dobutamine at $3 \mu \mathrm{g} / \mathrm{kg} /$ min and norepinephrine at $0.1 \mu \mathrm{g} / \mathrm{kg} / \mathrm{min}$ was started to maintain hemodynamic stability. Post-operative chest radiograph showed improvement in the size of the cardiac shadow with no pneumothorax or pneumopericardium. Her post-operative electrocardiograph (ECG) did not show signs of ischemia. However, post-operative transthoracic 2D-echo showed multisegmental wall hypokinesia with an ejection fraction (EF) of $38 \%$ by Simpson's method (Fig. 2). The patient was then admitted in the intensive care unit (ICU) with continuous hemo-

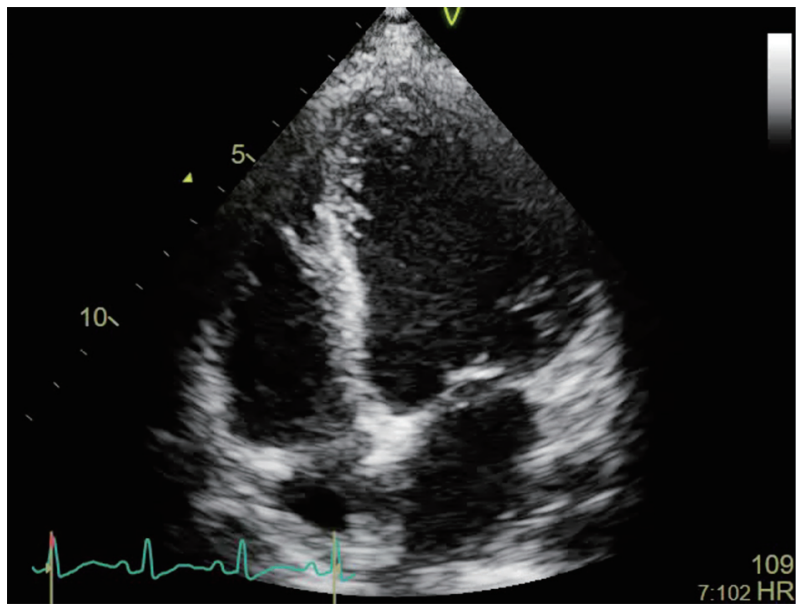

Figure 2. Post-pericardiostomy transthoracic 2D-echo (apical 4C view) showing minimal pericardial effusion but with a dilated left ventricular cavity with multi-segmental wall hypokinesia.

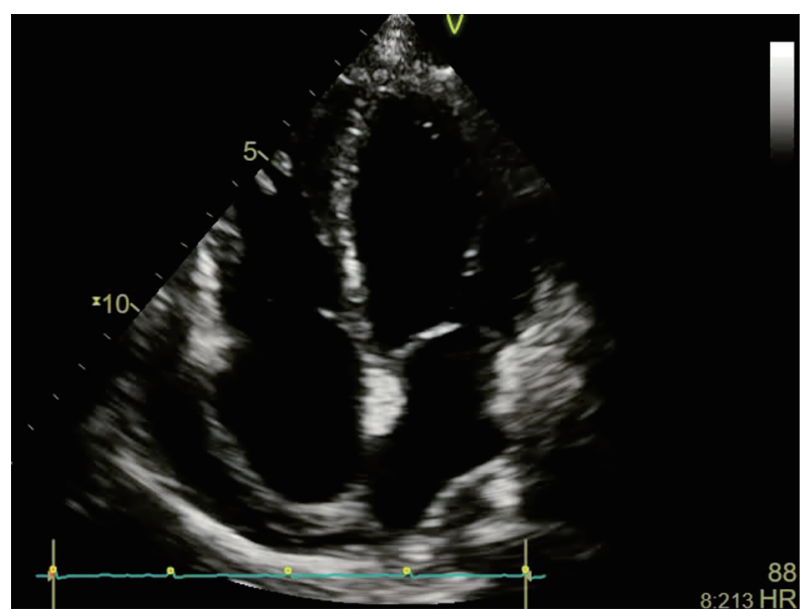

Figure 3. Transthoracic 2D-echo (apical 4C view) done after 3 days showing re-accumulation of pericardial effusion but with marked improvement in left ventricular function.

dynamic monitoring. She was admitted with inotropic support for $48 \mathrm{~h}$ and adequate fluid hydration. She was started on digoxin $0.25 \mathrm{mg}$ OD. On her third post-operative day, her hemodynamics improved significantly. The patient was weaned off from inotropic support and on repeat 2D transthoracic echo (Fig. 3), and there was marked improvement in RV and left ventricular (LV) systolic function. The patient had improved functionality and was discharged 5 days after the pericardial drainage.

\section{Discussion}

PDS was a term suggested in 2010 by Angouras et al. It is a syndrome of acute cardiac failure leading to hemodynamic deterioration with or without pulmonary edema developing after pericardial fluid drainage [1]. This syndrome has been reported differently in case reports and it can be associated with pericardiocentesis but more commonly with surgical fluid drainage. The clinical presentation varies in literature. Pradhan et al [2] published an analysis of reported cases from 1983 to 2013 among which 35 cases of PDS with different clinical manifestations occurred after pericardial fluid drainage (18 cases of pericardiocentesis, 16 cases of pericardiostomy and one both). In the paper, the onset of the LV dysfunction ranged from immediately after the procedure to after $48 \mathrm{~h}$. Cardiogenic shock from LV failure was the most common presentation (40\%) and around one-third presented with pulmonary edema without shock. Around $20 \%$ presented with biventricular failure with shock which was the clinical presentation of the case of this patient. The authors reported a high mortality rate of $30 \%$ with more patients died after surgical pericardial fluid drainage. A possible explanation is that the surgical removal of pericardial fluid led to a brisk increase in preload leading to rapid expansion of the RV [2].

Majority of cases of PDS do not have a pathophysiological mechanism to explain the paradoxical ventricular dysfunction. However, there are several mechanisms proposed such as 
autonomic dysfunction, hemodynamic overload, and ischemia (diminished coronary perfusion) hypothesis. According to the autonomic dysfunction hypothesis, there is an imbalance in the sympathetic-parasympathetic system with a possible reduction of sympathetic stimulation following the removal of the cardiac tamponade physiology which may exacerbate a pre-existing LV and/or RV dysfunction [3]. There may also be an underlying coronary ischemia from increased coronary vascular resistance caused by prolonged increased pericardial pressure. This mechanical pressure on the coronary arteries may lead to myocardial stunning and hibernation which can lead to a transient systolic dysfunction [4]. The most common explanation is related to the interventricular interdependence. There is a preload-afterload mismatch after rapid drainage of pericardial fluid which leads to sudden increase in venous return and sudden expansion of right chambers that occurs at the expense of the left chambers leading to a possible acute left-side heart failure and pulmonary edema [3-7].

In addition to the proposed mechanisms mentioned, in some of the patients there is already an underlying subclinical cardiac pathology that predisposes them to develop PDS such as cardiomyopathy from malignant myocardial infiltration or chemotherapy induced [6, 8-10].

Treatment of the syndrome is supportive and recovery of ventricular function is expected. In the case presented, recovery of LV function occurred after $72 \mathrm{~h}$ of supportive therapy comprising of inotropic support, adequate hydration, and continuous hemodynamic monitoring. There is no available literature on how to prevent this syndrome. Up to the present, there is still no recommended minimal amount of fluid for drainage in order to prevent this syndrome. In case reports it may occur even following the drainage of $<500 \mathrm{~mL}$ of pericardial fluid [5-7]. A suggested approach is to monitor the patient's hemodynamics via right heart catheterization or echocardiography while removing the pericardial fluid. Once there is evidence of improvement of the cardiac tamponade the removal of additional fluid is halted and a pericardial drainage tube is maintained to slowly remove the remaining fluid. Prolonged pericardial drainage can be removed when the daily fluid output is less than $30 \mathrm{~mL}[11,12]$.

\section{Conclusions}

We report this uncommon case since this syndrome is reported in literature using different terms such as pericardial drainage complicated by "low output cardiac syndrome", "ventricular dysfunction or failure", and "pulmonary edema". We recommend the acknowledgement of a uniform medical diagnosis so that this syndrome can be widely recognized and more effectively prevented and treated.

\section{Acknowledgments}

I cannot express enough thanks to my co-authors and consultant mentors for their continued support and guidance in the completion of this research. Also, this would not have been easily accomplished without the support of the Division of Cardiovascular Medicine and the Department of Medicine.

\section{Financial Disclosure}

None to declare.

\section{Conflict of Interest}

None to declare.

\section{Informed Consent}

An informed consent was signed by the patient prior to the publication of this study.

\section{Author Contributions}

All authors have directly participated in the writing of this study and have read and approved the final version submitted.

\section{Data Availability}

The data used to support the findings of this study are included within the article. Additional data or information can be requested by contacting the corresponding author.

\section{References}

1. Angouras DC, Dosios T. Pericardial decompression syndrome: a term for a well-defined but rather underreported complication of pericardial drainage. Ann Thorac Surg. 2010;89(5):1702-1703; author reply 1703.

2. Pradhan R, Okabe T, Yoshida K, Angouras DC, DeCaro MV, Marhefka GD. Patient characteristics and predictors of mortality associated with pericardial decompression syndrome: a comprehensive analysis of published cases. Eur Heart J Acute Cardiovasc Care. 2015;4(2):113-120.

3. Wolfe MW, Edelman ER. Transient systolic dysfunction after relief of cardiac tamponade. Ann Intern Med. 1993;119(1):42-44.

4. Skalidis EI, Kochiadakis GE, Chrysostomakis SI, Igoumenidis NE, Manios EG, Vardas PE. Effect of pericardial pressure on human coronary circulation. Chest. 2000;117(3):910-912.

5. Basmaji SG, Peretz-Larochelle M, Bernier ML. Pericardial decompression syndrome: a rare and potentially dramatic complication of pericardiocentesis. Int J Cardiol. 2015;178:297-298.

6. Vandyke WH, Jr., Cure J, Chakko CS, Gheorghiade M. Pulmonary edema after pericardiocentesis for cardiac 
tamponade. N Engl J Med. 1983;309(10):595-596.

7. Pulmonary edema after pericardiocentesis. N Engl J Med. 1984;310(6):391.

8. Karamichalis JM, Gursky A, Valaulikar G, Pate JW, Weiman DS. Acute pulmonary edema after pericardial drainage for cardiac tamponade. Ann Thorac Surg. 2009;88(2):675-677.

9. Ligero C, Leta R, Bayes-Genis A. Transient biventricular dysfunction following pericardiocentesis. Eur J Heart Fail. 2006;8(1):102-104.
10. Dosios T, Theakos N, Angouras D, Asimacopoulos P. Risk factors affecting the survival of patients with pericardial effusion submitted to subxiphoid pericardiostomy. Chest. 2003;124(1):242-246.

11. Imazio M, Mayosi BM, Brucato A, Markel G, Trinchero $\mathrm{R}$, Spodick DH, Adler Y. Triage and management of pericardial effusion. J Cardiovasc Med (Hagerstown). 2010;11(12):928-935.

12. Imazio M, Adler Y. Management of pericardial effusion. Eur Heart J. 2013;34(16):1186-1197. 